\title{
A footprint technique to identify white rhino Ceratotherium simum at individual and species levels
}

\author{
Sky K. Alibhai ${ }^{1}$, Zoe C. Jewell ${ }^{1, *}$, Peter R. Law $^{2}$ \\ ${ }^{1}$ Apartado 210, 8550-909 Monchique, Portugal \\ ${ }^{2} 1$ Mack Place, Monroe, New York 10950, USA
}

\begin{abstract}
A non-invasive and cost-effective footprint identification technique (FIT) is presented, which can aid the identification of individual white rhino Ceratotherium simum and the differentiation of this species from black rhino Diceros bicornis. FIT is an adaptation of a traditional tracking identification technique and is a useful censusing and monitoring tool for wildlife conservation. We implemented FIT to identify 40 white rhino. Geometric profiles were extracted from digital images of footprints, and subjected to an algorithm based on multivariate statistical analyses. FIT's classification rules were tested using a dataset of 1276 footprints from 159 tracks of 40 white rhino from a fenced wild population in Namibia. Using 2 different test models for pairwise track matching, FIT gave accuracies of 91 and $95 \%$ for population estimate (census) prediction. In a monitoring scenario (matching a 'test' track to one of the footprint sets of known individuals) the accuracies for 2 test models were 97 and $99 \%$. For species discrimination, we used a dataset of 1636 footprints, with 218 tracks of which 59 were from black and 159 from white rhino. FIT gave a species discrimination accuracy for tracks of 98 to $99 \%$ using 3 different test models. We outline how the underlying FIT has been adapted for white rhino and detail work in progress to extend the method to other species. We anticipate that the technique will offer an objective and accurate tool for monitoring and censusing, with flexibility as regards target species and locale. Data collection for FIT is intuitive for skilled trackers and thus local expertise can be employed. The technique promises to be an effective tool for management and ecological studies, especially for nocturnal or otherwise elusive species, and is expected to be effective as a complementary tool for other monitoring techniques, such as mark-recapture or camera-trapping.
\end{abstract}

KEY WORDS: Footprint · Non-invasive $\cdot$ Monitoring $\cdot$ Censusing $\cdot$ White rhino $\cdot$ Tracking

\section{INTRODUCTION}

Many facets of wildlife research demand the recognition of individual animals. Recognition of individuals has traditionally relied upon natural features of the species in question to aid human and, more recently, computer recognition abilities, e.g. coat patterns, such as tiger stripes (Karanth 1995, Karanth \& Nichols 1998) and cheetah spots (Kelly 2001) and other idiosyncratic characteristics such as lion (Pennycuick \& Rudnai 1970) and leopard (Miththapala et al. 1989) muzzle vibrissae spots, wrinkle patterns on rhinoceros snouts (Goddard 1966, Mukinya 1973), shape of whale flukes (Katona et al. 1979), and spots on certain sharks (Arzoumanian et al. 2005, van Tienhoven et al. 2007).
In the absence of such identifying features, or due to doubts regarding their reliability, biologists have resorted to artificial markings such as bands and radio transmitters. In recent years, more attention has been given to possible effects of 'tagging' animals (Murray \& Fuller 2000) and a general impetus to avoid costs to an animal associated with tagging and handling. Research on black rhino Diceros bicornis suggested an association between routine immobilisation for invasive monitoring methods (i.e. radio-collaring) and compromise of female fertility (Alibhai et al. 2001, Alibhai \& Jewell 2002) and also highlighted the unacceptably high failure rate of radio collars on black rhinoceros, as well as associated problems (Alibhai \& Jewell 2001). Moorhouse \& MacDonald (2005) pro- 
vided a brief literature review regarding the possible adverse effects of radio collaring which indicated that concern about such effects is not unwarranted. Murray \& Fuller (2000, p. 43) asserted of natural markings '...where the use of this technique meets study requirements, it is more desirable than artificial marking'.

Natural markings are, therefore, an attractive option when available, as they require no handling of individuals; however, subjectivity of pattern recognition is a potential problem (see Pennycuick \& Rudnai 1970 for an estimate of reliability of their pattern recognition technique). Moreover, unlike radio/GPS tagged individuals, the pattern-recognition method requires visual sighting and resighting of animals, which may be extremely difficult and fleeting for populations occurring at low density (either naturally or because endangered) and/or inhabiting terrain which makes locating and observing individuals difficult, or because the study animals are reclusive by nature or in response to human interference. Additionally, both tagging and the use of natural markings may impose nontrivial demands on research and/or management budgets.

For species which inhabit a landscape in which individuals regularly produce clear footprints and whose foot is of sufficient complexity to create a footprint with individual characteristics, tracks may serve as an alternative for natural marks, having the additional benefit that locating tracks is generally easier than locating individuals themselves. While this approach poses challenges, we think it offers considerable promise and is therefore very worthwhile to pursue for appropriate species and habitats.

Traditional trackers of many indigenous cultures are renowned for their ability not only to follow a track (an unbroken series of footprints) but also read information from it. If tracks are to become a useful scientific tool, the information must be extracted in an objective and repeatable fashion. Strangely, tracks have received relatively little attention from scientists (but see Stander et al. 1997). In Jewell et al. (2001), we briefly reviewed early attempts to identify Asian rhinoceroses from their footprints. While offering promise, these attempts tended to involve substantial subjectivity, were restricted by technological limitations on the methods for converting a footprint into data, and were rarely objectively tested.

Similar problems have been encountered when using tracks in tiger censuses, although some effort has been made to address these issues; see Sharma et al. (2005) for a recent review and a method that attempts to address the critique of Karanth et al. (2003). Some other attempts to introduce objective identification techniques for felids have been reported in the literature (e.g. Riordan 1998, Grigione et al. 1999) but were tested only in artificial circumstances. Similarly, L. Van
Bommel et al. (unpubl. data) successfully discriminated between captive lions using their footprints. Herzog et al. (2007) report a finger-printing approach for identifying individual fishers Martes pennanti from footprints made on track plates.

In Jewell et al. (2001), we described a method for identifying individual black rhino from their tracks using an objective procedure which yielded high reliability in tests. In the light of further experience, we have substantially refined this method, using a customised script JMP rather than SAS software, and employing modified statistical procedures described below. We now call it the Footprint Identification Technique (FIT). The method consists of 2 stages: (1) the capture of footprints and conversion into a geometric profile that serves as the data; (2) analyses to which the data are submitted for the purpose of classification. Digital cameras and computer software have made the first stage far more tractable than in early attempts. Such technology was already utilized in Jewell et al. (2001), and subsequent refinements to Stage 1 (ongoing) focus on automating digital-image processing. Most of the changes reported here for the white rhino Ceratotherium simum concern the analyses of Stage 2. The conceptual differences between FIT and Sharma et al. (2005) occur mostly in the analyses performed, but also in that FIT uses only the more objective digital images of footprints, rather than tracings.

We believe FIT may be effective for a variety of species in a range of habitats and is thus a valuable tool for estimating population abundance, monitoring endangered populations, and for ecological and behavioural studies.

\section{STUDY POPULATION}

\section{White rhino population status}

The Southern sub-species of the white rhino Ceratotherium simum simum is the least endangered of the rhinoceros subspecies (classified as Near-Threatened on the IUCN's Red List of Threatened Species, downlisted to CITES Appendix 2 from Appendix 1 in 1994), with a total estimated wild population as of 31 December 2005 of 14550 (Emslie et al. 2007, see also Amin et al. 2006). The population declined at the end of the 19 th century to very low numbers, though exactly how low is subject to debate (Owen-Smith 1988, Emslie \& Brooks 2002, Rookmaker 2002). The protection and management of this species depends on effective censusing and monitoring. At the end of 2005, Namibia had the third largest population of white rhino after the Republic of South Africa and Zimbabwe, with an estimated population of 293 individuals (Emslie et al. 2007). 


\section{Study area and white rhino population}

In order to implement FIT and validate the resulting algorithm, a test population was required. The Namibian Ministry of Environment and Tourism suggested the Otjiwa Game Ranch in Namibia, a fenced area of approximately $100 \mathrm{~km}^{2}$ in central Namibia. Mean annual rainfall was around $500 \mathrm{~mm}$. Substantial areas of sandy substrate in open terrain and game trails through acacia woodland, combined with determined effort, made footprint collection feasible. The dominant vegetation was acacia.

The current white rhino population at Otjiwa was re-introduced during the early 1970s (Joubert 1996) and numbered 26 in 1999. Further individuals were added to the population in 2002 and 2003, bringing the total number of identified animals to 40 for the testing of FIT. Monitoring the population over this extended period of time, using a combination of visual identification techniques independently of FIT, provided reliable data on individual identifications, which were kept updated as animals were imported, or born into the population, or were exported or removed by death or poaching. Monitoring was undertaken mainly by a small anti-poaching unit (APU) with the assistance of visiting students and research teams. Otjiwa provided a good test population, as individual rhino were known, the site was fenced and the substrate was suitable.

\section{Footprint collection}

\section{Definition of footprint terms}

In a dataset of footprint images, collected from a given population over a fixed period of time, each footprint is part of a 'track' (an uninterrupted pathway made by 1 animal), and all the footprints available for each animal at the end of the study form a collection of such tracks constituting the 'set' for that animal. The total collection of sets available for all the animals in the study forms the 'library'. In the literature, footprints are also referred to as 'spoor', 'pug-marks' or 'pugs'. Table 1 shows gender, known age, numbers of footprints and numbers of original and modified tracks (see subsection 'FIT for Censusing: The Canonical Pairwise Comparison Technique (CPCT)') for the 40 rhino.

\section{Collection of footprints at the study site}

As in Jewell et al. (2001), many factors were found to influence footprint quality. Only fresh and undistorted left hind footprints showing good detail were used in the study (left hind or right hind can be equally used;
Table 1. Ceratotherium simum. Sex, age, number of footprint images, number of original tracks and number of modified tracks for each of the 40 white rhinos in the study; track size: footprints per track

\begin{tabular}{|c|c|c|c|c|c|}
\hline $\begin{array}{l}\text { Rhino } \\
\text { ID }\end{array}$ & Sex & $\begin{array}{l}\text { Age } \\
(\mathrm{yr})\end{array}$ & $\begin{array}{c}\text { No. of } \\
\text { footprints }\end{array}$ & $\begin{array}{c}\text { No. of } \\
\text { original tracks } \\
\text { (track size) }\end{array}$ & $\begin{array}{c}\text { No. of } \\
\text { modified tracks } \\
\text { (track size) }\end{array}$ \\
\hline 01 & $\mathrm{~F}$ & 00.25 & 10 & $2(4-6)$ & $1(8)$ \\
\hline 02 & $\mathrm{~F}$ & 00.25 & 36 & $5(6-10)$ & $5(6-8)$ \\
\hline 03 & $\mathrm{~F}$ & 00.50 & 13 & $2(6-7)$ & $2(6-7)$ \\
\hline 04 & $\mathrm{~F}$ & 01.95 & 21 & $2(8-9)$ & $2(8)$ \\
\hline 05 & $\mathrm{~F}$ & 02.00 & 16 & $2(5-11)$ & $2(8)$ \\
\hline 06 & $\mathrm{~F}$ & 02.25 & 19 & $2(8-11)$ & $2(8)$ \\
\hline 07 & $\mathrm{~F}$ & 02.50 & 19 & $2(9-10)$ & $2(8)$ \\
\hline 08 & $\mathrm{~F}$ & 02.65 & 47 & $6(3-9)$ & $6(7-8)$ \\
\hline 09 & $\mathrm{~F}$ & 03.75 & 30 & $4(7-8)$ & $4(7-8)$ \\
\hline 10 & $\mathrm{~F}$ & 06.00 & 58 & 7 (8) & $7(8)$ \\
\hline 11 & $\mathrm{~F}$ & 06.40 & 44 & $6(4-8)$ & $5(8)$ \\
\hline 12 & $\mathrm{~F}$ & 06.60 & 70 & $9(7-8)$ & $9(7-8)$ \\
\hline 13 & $\mathrm{~F}$ & 07.50 & 21 & $3(7)$ & $3(7)$ \\
\hline 14 & $\mathrm{~F}$ & 08.50 & 32 & $4(7-9)$ & $4(8)$ \\
\hline 15 & $\mathrm{~F}$ & $10.00+$ & 21 & $2(9-12)$ & $2(8)$ \\
\hline 16 & $\mathrm{~F}$ & $10.00+$ & 41 & $5(6-9)$ & $5(6-8)$ \\
\hline 17 & $\mathrm{~F}$ & $10.00+$ & 22 & $4(5-6)$ & $3(7-8)$ \\
\hline 18 & $\mathrm{~F}$ & $10.00+$ & 27 & $3(8-10)$ & $3(8)$ \\
\hline 19 & $\mathrm{~F}$ & $10.00+$ & 49 & $5(9-11)$ & $5(8)$ \\
\hline 20 & $\mathrm{~F}$ & $10.00+$ & 42 & $5(6-11)$ & $5(7-8)$ \\
\hline 21 & $\mathrm{M}$ & 00.25 & 16 & $3(5-6)$ & $2(8)$ \\
\hline 22 & $\mathrm{M}$ & 00.33 & 31 & $4(7-11)$ & $4(7-8)$ \\
\hline 23 & $\mathrm{M}$ & 00.33 & 11 & $2(4-7)$ & $1(8)$ \\
\hline 24 & $\mathrm{M}$ & 00.85 & 15 & $2(7-8)$ & $2(7-8)$ \\
\hline 25 & $\mathrm{M}$ & 00.95 & 10 & $1(10)$ & $1(8)$ \\
\hline 26 & $\mathrm{M}$ & 01.25 & 37 & $5(3-9)$ & $5(6-8)$ \\
\hline 27 & $\mathrm{M}$ & 01.33 & 11 & $2(3-8)$ & $1(8)$ \\
\hline 28 & M & 01.60 & 16 & $2(8)$ & $2(8)$ \\
\hline 29 & M & 02.20 & 37 & $5(5-8)$ & $4(7-8)$ \\
\hline 30 & M & 02.75 & 39 & $5(6-8)$ & $5(6-8)$ \\
\hline 31 & $\mathrm{M}$ & 04.40 & 29 & $4(6-9)$ & $4(7-8)$ \\
\hline 32 & M & 04.50 & 42 & $5(7-9)$ & $5(7-8)$ \\
\hline 33 & M & 05.50 & 40 & $5(6-9)$ & $5(6-8)$ \\
\hline 34 & M & 06.00 & 31 & $4(6-9)$ & $4(6-8)$ \\
\hline 35 & M & 06.00 & 37 & $4(9-10)$ & $4(8)$ \\
\hline 36 & M & 07.50 & 47 & $5(8-10)$ & $5(8)$ \\
\hline 37 & M & 08.00 & 14 & $2(6-8)$ & $2(6-8)$ \\
\hline 38 & M & 08.50 & 35 & $4(8-9)$ & $4(8)$ \\
\hline 39 & M & $10.00+$ & 27 & $3(8-10)$ & $3(8)$ \\
\hline 40 & M & $10.00+$ & 108 & $12(6-12)$ & $12(6-8)$ \\
\hline \multicolumn{6}{|l|}{ Total } \\
\hline 40 & $20 \mathrm{M} / 20 \mathrm{~F}$ & & 1276 & $159(3-12)$ & $152(6-8)$ \\
\hline
\end{tabular}


we use left hind as a standard, and hind rather than front because for many species, hind feet usually overstep the front feet when the individual is walking). The location of every track photographed was recorded with GPS. We further avoided the risk of collecting a footprint more than once by obliterating each after photography, and, by aging prints accurately, collecting only those which were fresh.

During the study periods 1999, 2002 and 2003, each animal was tracked, and digital images of its footprints were collected. Particular care was taken in situations where several rhino were found together to ensure that footprints were collected from the target animal only, typically achieved by backtracking (using only uninterrupted tracks) from a point at which the animal had been seen to place its foot.

Fresh footprints were also collected from the area immediately around waterholes and tracked until the rhino was located. We assumed that before the rainy season each rhino would have to visit a waterhole at least every other day (see Owen-Smith 1988) and that if all waterholes could be covered on a regular basis, footprints from each rhino would be collected. Initially each of the 28 waterholes was inspected at least once every $3 \mathrm{~d}$, to ascertain rhino usage. The ratio of number of visits to number of fresh footprints found was then calculated, and waterholes subsequently inspected according to rhino usage.

Additionally, all roads, including boundary roads, were mapped and each driven at least once a week and again, fresh footprints were either identified as known or tracked to the animal.

\section{Population library}

Since each animal had been unambiguously identified, regular tracking and observation provided distinctive tracks from 40 ind. The resulting library consisted of 1276 footprints and 159 original tracks from 40 white rhino (Table 1). For any one individual, only tracks for a single survey period, usually lasting $3 \mathrm{mo}$, were used. Table 1 shows the composition of the population. For the black rhino, data from previous studies in Zimbabwe (Jewell et al. 2001) were used.

\section{FIT METHODOLOGY}

\section{Determining the measurements to be taken from the footprint; creating a geometric profile}

The white rhino (Order: Perissodactyla) has 3 toes on each foot. The distal (third) phalanx of each digit is enclosed in a horny hoof. The plantar cushion helps support the distal metatarsals and digits where they make ground contact. Fig. 1a shows the sole of a white rhino foot. The impression made by the foot can reveal clear outlines of the outside edge of each hoof, and also the outline of the hind part, or heel, of the plantar cushion.

Details of the photographic technique and preprocessing of the footprint prior to statistical analyses were predominantly the same as those in Jewell et al. (2001). The main difference in the current approach was that we employed JMP Statistical Discovery Software (SAS) not only for all statistical procedures but for the majority of the preprocessing of the footprint prior to statistical analysis, as explained below.

Each implementation of FIT to a new species requires the choice of a set of natural landmarks determined by the structure of the footprint. Once the footprint image is taken it is imported into Adobe Photoshop software where it is photo-optimised, image orientation standardised by rotation, and the landmark points manually placed using cross-hair guidelines to minimise bias. The landmark points are selected on the basis of foot anatomy to include those points which are clearly definable and repeatable across many footprints. After placing these landmark points, a set of derived landmarks, geometrically constructed from the set of natural landmarks, is then defined. The derived points are positioned by JMP script into which their geometrical constructions have been entered (see Fig. 1b). The full set of points is designed to allow all measurements that one anticipates might prove useful in discriminating between footprints. Table 2 provides the descriptions of the landmark and derived points and Table 3 lists the 113 measurements (distances and angles) generated in JMP. The resulting set of measurements constitutes the geometric profile of the footprint image, and provides the data upon which all FIT analyses are performed.

\section{Determining which measurements best discriminate; variable selection}

The geometric profile typically contains many measurements $(>100$ in the application of FIT to African rhino), and the first step in FIT analysis must be the reduction of the total number of measurements to the set (referred to as the FIT algorithm) which provides good discrimination, whether between individuals or species. For this purpose, a library of footprints from known individuals is required. The measurements for each footprint in the given library are labelled by in dividual identity, and these data are entered into the discriminant analysis (DA) module of 'JMP from SAS software', with the stepwise option checked. The for- 


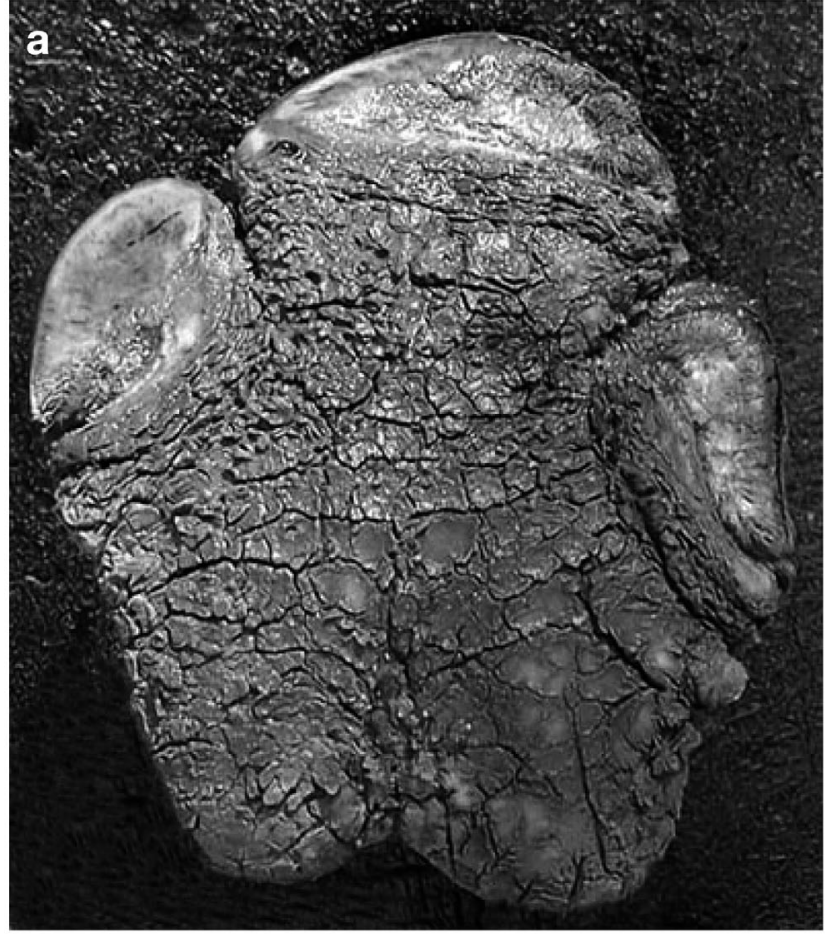

Fig. 1. Ceratotherium simum. (a) White rhino left hind foot, showing the plantar cushion and 3 toes. (b) White rhino left hind footprint showing landmark points $(O)$ and derived points (ם) with their respective numbers. The distance between the 2 points marked on the vertical scale is $20 \mathrm{~cm}$

ward stepwise procedure is then employed to select those measurements which will produce an effective classification for FIT analyses.

Note that no hypothesis is framed or statistical test performed here, only a selection of measurements. The deficiencies of stepwise variable selection in the construction of explanatory causal models are well known (Whitaker 1997). As we are not constructing an explanatory model, we adopt this procedure here as a simple objective routine to select variables. We make no assumption that this approach selects the subset of variables with the greatest discriminatory power, only that it selects a subset which, in the validation tests, results in a high degree of discrimination in the FIT analyses.

\section{Using the measurements to discriminate; FIT analyses}

There are 2 extreme cases which FIT analyses address with regard to classification of individual tracks within the species: (1) individuals are unknown and have to be identified on the basis of collected tracks, and (2) individuals are known and a new obser-

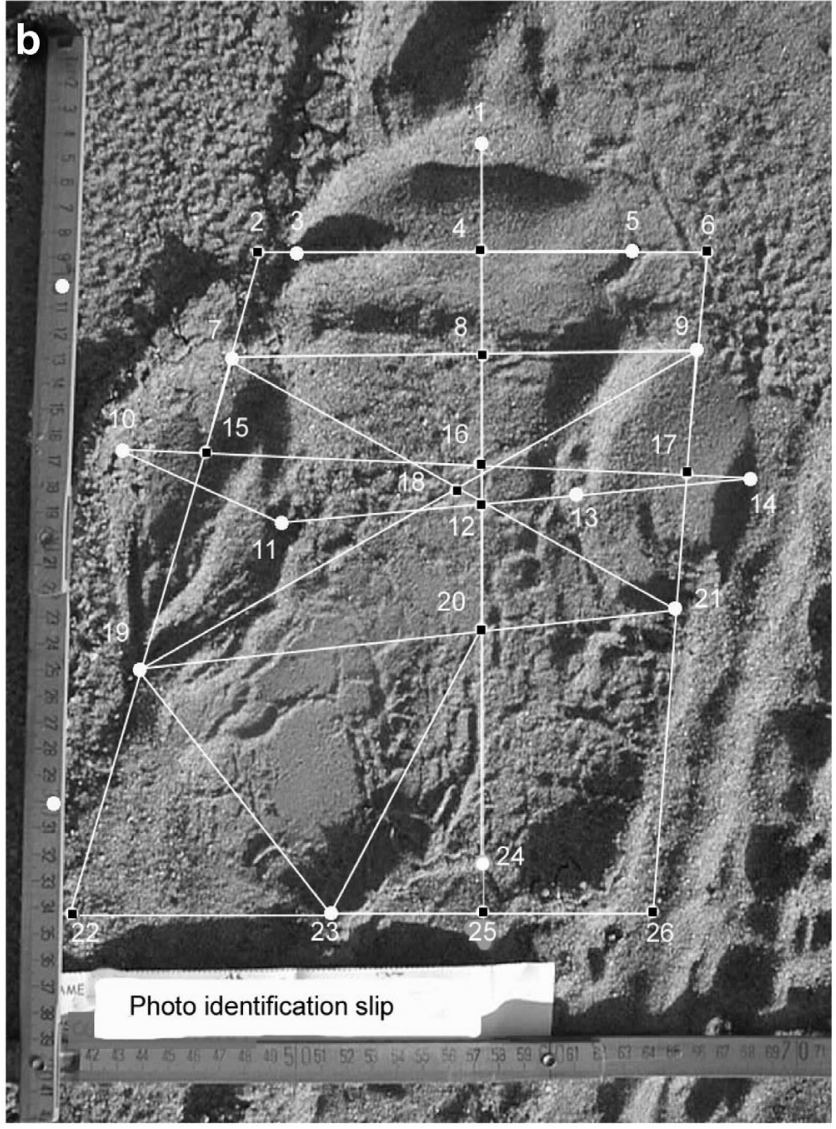

vation (track) is to be assigned to one of the known individuals. For convenience, we will refer to the first scenario as population 'censusing' (i.e. estimating the abundance of an unknown population, Caughley \& Sinclair 1994) and to the second as 'monitoring'.

Population monitoring invites the application of DA. Classificatory DA (e.g. Johnson \& Wichern 1998) provides a classification scheme which is optimal with regard to minimising probability of misclassification. Posing theoretical probability distributions for the geometric profile of the footprints of each individual in a population seems unrealistic, however, as does, perhaps, the assumption that they are likely to be normally distributed with the same covariance matrix (the typical assumption required to extract a tractable and explicit discriminant score for classification). In practice, given a library of footprints belonging to known individuals, one could submit a new observation to a classificatory DA scheme. For example, JMP assigns a new observation to the group whose mean it is closest to in Mahalanobis distance, assuming the groups have the same covariance matrix; this classification scheme is known to coincide with the optimal classification scheme of classificatory DA when the groups are also normally distributed (assuming also equal prior 
Table 2. Definitions of landmark point positions placed on white rhino left hind footprint images. L: line; EL: extension of a line; e.g. L1-2: line connecting point nos. 1 and 2 (points as in Fig. 1b)

\begin{tabular}{|ll|}
\hline Point no. & Definition of position on left hind foot \\
\hline \multicolumn{2}{l}{ Landmark point positions } \\
1 & Highest point of front toe \\
3 & Most lateral point of front toe \\
5 & Most medial point of front toe \\
7 & Highest point of lateral toe \\
9 & Highest point of medial toe \\
10 & Most lateral point of lateral toe \\
11 & Most medial point of lateral toe \\
13 & Most medial point of medial toe (nearest \\
& midline of foot) \\
14 & Most lateral point of medial toe (furthest \\
& from midline of foot) \\
21 & Lowest point of lateral toe \\
23 & Lowest point of medial toe \\
24 & Lowest point of heel \\
& Point where perpendicular to L7-9 \\
Derived point & positions \\
2 & Intersection (EL5,3) with (EL19,7) \\
4 & Intersection (L1,24) with (L3,5) \\
6 & Intersection (EL3,5) with (EL21-9) \\
8 & Intersection (L1-24) with (L7-9) \\
12 & Intersection (L1-24) with (L11-13) \\
15 & Intersection (L7-19) with (10-14) \\
16 & Intersection (L1-24) with (L10-14) \\
17 & Intersection (L9-21) with (L10-14) \\
18 & Intersection (L7-21) with (L9-19) \\
20 & Intersection (L1-24) with (L19-21) \\
25 & Extension of (L1-24) to create 25, where \\
22 & IL23-25) parallel to (L7-9) \\
26 & Intersection (EL7-19) with (EL25-23) \\
& Intersection (EL9-21) with (EL23-25) \\
& \\
&
\end{tabular}

probabilities of group membership and equal costs of misclassification, and setting aside the fact that in practice sample estimates of group means and covariances must be employed). We reported the results of such an exercise in Jewell et al. (2001) for the black rhino of that study (and repeated the exercise with the current dataset with similar results) but have decided not to include this approach to monitoring as part of FIT for individual classification.

Rather than view classification of footprints as an issue of trying to force data to fit theoretical ideals (which, if feasible, would have the attraction of allowing actual inference of group membership), we see the problem of footprint identification more as one of 'pattern recognition', i.e. contriving algorithms which, using training data, are trained to match that data to their known identities. The resulting algorithms must then be validated on test data.

The algorithms in FIT are all based on the canonical variates of Fisher's approach to DA (see Mardia et al. 1979). The canonical variates require no distributional assumptions to derive and are the linear combinations of the observed variables that best separate group means. They are, therefore, natural candidates for the ingredients of the FIT algorithms. One must, however, assume common group covariances in order to interpret their statistical properties; in particular, to deduce that Euclidean distance (upon which classification in canonical space is based) is equivalent to Mahalanobis distance in the original data space. If the groups are further assumed to be normally distributed, classification in canonical space is equivalent to standard classificatory DA (see Williams 1982). Without the assumption of common covariance, we cannot infer that canonical space does not distort Mahalanobis distance in data space; even if this assumption were granted, without normality, classification in canonical space may be suboptimal; moreover, if one does not exploit all available canonical variates, use of these for statistical classification is certainly less than optimal. In line with the argument of the previous paragraph, rather than making dubious statistical arguments, we simply appeal to Fisher's argument that the canonical variates are useful for separating group means and seek to formulate algorithms in terms of those that work successfully on test data.

For discriminating between species, however, as there are only 2 'groups' there is only a single canonical variate and in this case we do resort to linear DA (LDA). In this case, the differences between individuals are expected to average out and it is not perhaps unreasonable to suppose that the data from the 2 species of rhino will conform to the assumptions of LDA.

Because an isolated footprint might too readily constitute an outlier, we use only individual tracks and amalgams/splitting of tracks in all cases.

FIT for censusing; the Canonical Pairwise Comparison Technique (CPCT)

In this case, the study population is unknown. Measurement selection must be carried out using a test population. The test population may be a captive population or a wild population from another study site. In the census scenario, footprints (as tracks) are collected from an unknown population to form a library of footprints in tracks without set structure. The objective is to assign the correct set structure to the library. Note that this problem is not addressed by DA. The FIT algorithm is again based, however, on canonical variates. The idea is to make pairwise comparisons of tracks and decide whether or not they belong to the same individual.

For each pair of tracks, one forms the 2 groups consisting of the footprints in each of these tracks and, additionally, a third group, the Reference Centroid 
Table 3. Definitions of 113 measurements from each left hind footprint image of white rhino generated in JMP using landmark and derived points as reference. Measurements M1-47 show length (e.g. L2-7: length from point 2 to 7 ), measurements M48-77 the angle (e.g. A7, 1, 8: angle formed between intersection L7-1 and L8-1), and combined measurements CM78-113 the combined lengths and angles (e.g. M1-4 is measurements 1 to 4 inclusive). For points see Fig. $1 \mathrm{~b}$

\begin{tabular}{|c|c|c|c|c|c|}
\hline M1-47 & Definition & M48-77 & Definition & CM78-113 & 3 Definition \\
\hline M1 & L2-7 & M48 & $\mathrm{A} 7,1,8$ & CM78 & M1-4 \\
\hline M2 & L7-15 & M49 & $\mathrm{A} 8,1,9$ & CM79 & M2-3 \\
\hline M3 & L15-19 & M50 & $\mathrm{A} 7,2,3$ & CM80 & M5-6 \\
\hline M4 & L19-22 & M51 & $\mathrm{A} 8,3,4$ & CM81 & M5-7 \\
\hline M5 & L1-4 & M52 & $\mathrm{A} 4,5,8$ & CM82 & M5-8 \\
\hline M6 & L4-8 & M53 & A5 6,9 & CM83 & M5-9 \\
\hline M7 & L8-16 & M54 & $\mathrm{A} 7,4,8$ & CM84 & M5-10 \\
\hline M8 & L16-20 & M55 & $\mathrm{A} 8,4,9$ & CM85 & M6-7 \\
\hline M9 & L20-24 & M56 & $\mathrm{A} 8,7,4$ & CM86 & M6-8 \\
\hline M10 & L24-25 & M57 & $\mathrm{A} 4,9,8$ & CM87 & M6-9 \\
\hline M11 & L6-9 & M58 & $\mathrm{A} 18,7,8$ & CM88 & M6-10 \\
\hline M12 & L9-17 & M59 & $\mathrm{A} 18,9,8$ & CM89 & M9-10 \\
\hline M13 & L17-21 & M60 & $\mathrm{A} 15,7,18$ & CM90 & M11-14 \\
\hline M14 & L21-26 & M61 & $\mathrm{A} 18,9,17$ & CM91 & M12-13 \\
\hline M15 & L2-3 & M62 & $\mathrm{A} 16,15,7$ & CM92 & M15-16 \\
\hline M16 & L3-4 & M63 & $\mathrm{A} 16,17,9$ & CM93 & M15-18 \\
\hline M17 & L4-5 & M64 & $\mathrm{A} 13,12,8$ & CM94 & M16-17 \\
\hline M18 & L5-6 & M65 & $\mathrm{A} 19,18,21$ & CM95 & M17-18 \\
\hline M19 & L7-8 & M66 & $\mathrm{A} 15,19,18$ & CM96 & M19-20 \\
\hline M20 & L8-9 & M67 & $\mathrm{A} 18,19,20$ & CM97 & M22-23 \\
\hline M21 & L10-11 & M68 & $\mathrm{A} 17,21,18$ & CM98 & M25-28 \\
\hline M22 & L11-12 & M69 & $\mathrm{A} 18,21,20$ & CM99 & M26-27 \\
\hline M23 & L12-13 & M70 & $\mathrm{A} 25,19,20$ & CM100 & M29-30 \\
\hline M24 & L13-14 & M71 & $\mathrm{A} 23,19,25$ & CM101 & M31-33 \\
\hline M25 & L10-15 & M72 & A22, 19,23 & CM102 & M32-33 \\
\hline M26 & L15-16 & M73 & $\mathrm{A} 23,21,20$ & CM103 & M38-39 \\
\hline M27 & L16-17 & M74 & A $25,21,23$ & CM104 & M40-41 \\
\hline M28 & L17-14 & M75 & A26, 21, 25 & CM105 & M48-49 \\
\hline M29 & L19-20 & M76 & $\mathrm{A} 23,22,19$ & CM106 & M54-55 \\
\hline M30 & L20-21 & M77 & A25, 26, 21 & CM107 & $\mathrm{M} 56+58+60$ \\
\hline M31 & L22-23 & & & CM108 & M58-60 \\
\hline M32 & L23-25 & & & CM109 & M59-61 \\
\hline M33 & L25-26 & & & CM110 & M66-67 \\
\hline M34 & L7-1 & & & CM111 & M68-69 \\
\hline M35 & L1-9 & & & CM112 & M70-71 \\
\hline M36 & $\mathrm{L} 7-4$ & & & CM113 & M73-75 \\
\hline M37 & L4-9 & & & & \\
\hline M38 & L7-18 & & & & \\
\hline M39 & L18-21 & & & & \\
\hline M40 & L19-18 & & & & \\
\hline M41 & L18-9 & & & & \\
\hline M42 & L3-8 & & & & \\
\hline M43 & L8-5 & & & & \\
\hline M44 & L19-25 & & & & \\
\hline M45 & L25-21 & & & & \\
\hline M46 & L19-23 & & & & \\
\hline M47 & L23-21 & & & & \\
\hline
\end{tabular}

3 groups (note that the 2 tracks alone would provide only a single canonical variate) and inspecting the corresponding plot in canonical space of group means and $95 \%$ confidence regions. If the confidence regions of the 2 test tracks overlap, the tracks are said to be related. The library of tracks is partitioned into sets as follows: a set consists of a track, all the tracks related to it, all the tracks related to those tracks, and so on. In mathematical terms, this relatedness notion is taken to be an equivalence relation and the sets are the equivalence classes.

If each pair of tracks could be regarded as samples from multivariate normal distributions with common covariances, Hotelling's $T^{2}$-test could be used to determine whether 2 tracks were significantly different. As with the Canonical Ellipse Reduction Technique (CERT), however, in the absence of distributional assumptions, we have formulated a criterion for deciding whether 2 tracks are to be regarded as belonging to the same individual by the non statistical but unambiguous device of whether their $95 \%$ confidence regions overlap or not in a certain canonical space.

We tested the accuracy of CPCT for the white rhino population in 2 ways. Firstly, we extracted from the total footprint library the first pair of test tracks to be compared. The rest of the tracks in the library were used for algorithm development (by measurement selection) and combined to produce the RCV. Track sizes of 6 to 8 footprints were produced by arbitrary reduction or concatenation and are defined as modified tracks. The reasons for carrying out this procedure are described in Jewell et al. (2001) and we also deal with this in the 'Results' and 'Discussion'. Each time a comparison of 2 tracks was made using CPCT, a new algorithm consisting of 12 measurements selected using the forward stepwise feature was developed from the remainder of the library. Hence, each pairwise comparison resulted in the presence of 3 ellipses, 2 for the test tracks and one for the RCV. Classification accuracy was determined by the presence or absence of overlap of the $95 \%$ confidence interval ellipses for the 2 test tracks only. This tested both the

Value (RCV), consisting of all footprints in the library other than those belonging to the 2 tracks being tested (a library of tracks must be collected prior to analysis). The CPCT, already presented in Jewell et al. (2001), consists in computing the 2 canonical variates for these 'self' tracks (tracks known to belong to the same animal, in which case an ellipse overlap was the correct classification) and the 'non-self' tracks (tracks known to belong to different individuals, in which case a lack of overlap of ellipses was the correct classification). 
A second test was performed by arbitrarily allocating $50 \%$ of each set of tracks into algorithm and RCV construction. The remainder of the modified tracks were tested using the procedure described above. This test procedure resulted in 64 tracks from 35 ind. However, in this case, the 12 measurements selected and the RCV were derived from the data attributed to the algorithm and remained the same for all pairwise comparisons (see Table 4).

For the above procedures, the choice of 12 measurements was determined by testing between 6 and 15 measurements, all selected by the stepwise feature on the data to establish which number gave the most accurate track classification in pairwise interactions. Including more measurements increased separation of ellipses between tracks of the same individual, while reducing the number of measurements increased the likelihood of overlap between tracks from different individuals.
Once again we tested the efficacy of FIT using 2 models. In the first model, each of the 159 original tracks from the 40 ind. was considered as the test track with the remainder of the library employed for variable selection, so that a new set of variables was selected for each test. We tested the entire library for the relationship between the number of variables used and the percentage of footprints correctly classified by CERT. Fig. 2 shows an accuracy of $85 \%$ obtained with approximately 15 measurements, this accuracy reaching $92 \%$ with 30 variables. We used 30 variables since the analysis could be carried out in JMP just as expediently. In the second model, we arbitrarily allocated $50 \%$ of the footprints from each set to algorithm construction and the rest of the footprints (as tracks) were used for testing. This had the effect of reducing the number of test tracks because there were no self-tracks to compare against, since these had been assigned to

\section{FIT for monitoring; CERT}

In a monitoring context, one has a reference library of tracks from a known population of individuals. Using this library, measurements are selected as described in 'Determining which measurements best discriminate; variable selection'. Monitoring involves collecting new tracks from the known population and assigning them to one of the known identities.

The algorithm for assigning identity to a new track is provided by a new method developed since Jewell et al. (2001): CERT. Each known individual is represented by a set of footprints (consisting of all its tracks in the library) and the unknown track is tested against these. JMP computes the first 2 canonical variates for these data and produces the corresponding 2dimensional (canonical-space) plot of group means and $95 \%$ confidence ellipses. (Note that the number of canonical variates available is well known to be at most $\min (p, g-1)$, where $p$ is the number of variables involved in the analysis and $g$ the number of groups; in CERT more than 2 canonical variates would be available but we restrict to 2 because the canonical plot available in JMP was, until version 7.0, restricted to 2 dimensions.) All groups whose confidence ellipses do not overlap with the unknown track are set aside and the JMP procedure repeated. If this routine ultimately results in a plot of the unknown track overlapping exactly 1 group, that group's identity is assigned to the track. Otherwise the track remains unclassified.
Table 4. Number of measurements selected stepwise (measurement 01 selected first) used in the analyses for individual identification (first 12 measurements for CPCT and all 30 for CERT) and species identification (30 measurements). For all analyses, models based on $50 \%$ of the data used for model construction and $50 \%$ for testing. For explanation of measurements see Table 3

\begin{tabular}{|c|c|c|}
\hline $\begin{array}{l}\text { Sequence of } \\
\text { measurements } \\
\text { selected stepwise }\end{array}$ & $\begin{array}{l}\text { Individual identification } \\
\text { measurements }\end{array}$ & $\begin{array}{c}\text { Species identification } \\
\text { measurements } \\
\text { used for CERT }\end{array}$ \\
\hline 01 & CM98 & M34 \\
\hline 02 & M3 & M67 \\
\hline 03 & M65 & M24 \\
\hline 04 & M34 & CM94 \\
\hline 05 & CM97 & M2 \\
\hline 06 & M2 & M28 \\
\hline 07 & M40 & M25 \\
\hline 08 & M38 & M63 \\
\hline 09 & CM106 & M49 \\
\hline 10 & M63 & M53 \\
\hline 11 & M15 & M41 \\
\hline 12 & M24 & M76 \\
\hline 13 & CM94 & CM91 \\
\hline 14 & CM90 & M22 \\
\hline 15 & CM99 & M59 \\
\hline 16 & CM91 & CM79 \\
\hline 17 & CM100 & CM98 \\
\hline 18 & M39 & M64 \\
\hline 19 & M21 & M18 \\
\hline 20 & M53 & CM87 \\
\hline 21 & M58 & M58 \\
\hline 22 & M25 & M62 \\
\hline 23 & M6 & M66 \\
\hline 24 & CM108 & M7 \\
\hline 25 & M68 & M13 \\
\hline 26 & M77 & M65 \\
\hline 27 & M67 & M37 \\
\hline 28 & M62 & M54 \\
\hline 29 & M42 & CM97 \\
\hline 30 & M51 & M74 \\
\hline
\end{tabular}


the algorithm. Nonetheless, there were still 69 test tracks available, ranging in size from 3 to 11 footprints. For this model, the 30 measurements selected stepwise remained the same for all the test tracks (see Table 4).

FIT for species discrimination between the white and black rhino, using discriminant analysis

Using the original tracks (Table 1) we compared tracks from white rhino from the Otjiwa population (40 ind., 1276 footprints, 159 tracks) and black rhino from a population in the Sinamatella Intensive Protection Zone, Hwange National Park, Zimbabwe (18 individuals, 360 footprints, 59 tracks). In the first test model, a track was selected as test track, excluded from the dataset, and the remaining data subjected to linear discriminant analysis with species as the ' $x$ ' category and using 30 variables selected stepwise in JMP. JMP then gave the species classification prediction for this test track. This entire routine was repeated for each available track as test track, each time excluding the test track in question from the data used to select the variables. A track was deemed to have been classified correctly when a majority of the footprints in that track had the correct prediction. If there was a tie then the track was considered unclassified. In the second model, $50 \%$ of the footprints from each individual were assigned to algorithm construction and the rest of the footprints were tested as individual tracks.

To establish the number of variables to use, we once again tested the classification accuracy of individual

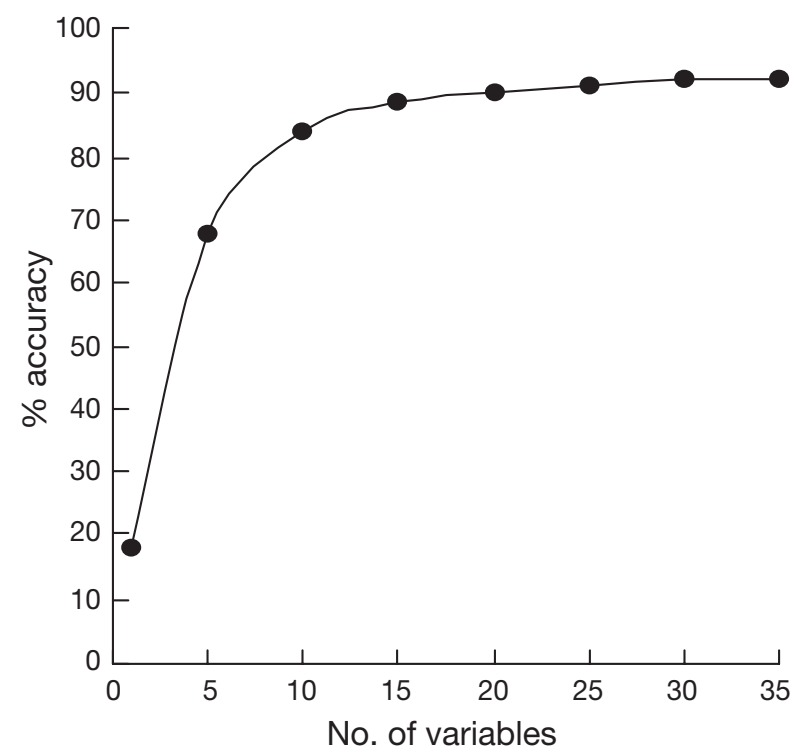

Fig. 2. Ceratotherium simum. Relationship between the number of variables (measurements) used in the analysis and the accuracy of footprint identification of footprints footprints using LDA and found 30 variables gave over $90 \%$ accuracy. We used 30 variables using the stepwise procedure in JMP for both models. Table 4 shows the 30 measurements used for the second model (50\% algorithm $/ 50 \%$ testing) in which the measurements were the same for all test tracks.

\section{RESULTS}

\section{The geometric profile}

The similarity between black and white rhino footprints indicated that the 13 natural landmark points for white rhino are the homologues of those for black rhino. Thus, the 13 derived landmark points were constructed exactly as for the black rhino (see Fig. 1b); and the total number of variables generated (113) was the same as that for the black rhino (Jewell et al. 2001).

\section{FIT for censusing; CPCT}

To test the accuracy of FIT for censusing, the CPCT was employed to assign identities to tracks, and these assignments were then compared to the known identities. Thus, as described in 'FIT methodology; FIT for censusing', paired tracks in the database were sequentially excluded from algorithm development for variable selection and tested for classification, resulting in 11329 pairwise interactions. A total of 104 interactions gave false classification, resulting in an accuracy of $99.1 \%$. Based on these interactions, we then grouped the tracks according to the matches to derive the predicted number of rhinos, which was 42 , giving a census accuracy of $95 \%$.

Using a different test, in which only $50 \%$ of footprints from each set were removed for algorithm development prior to testing (with the rest used as test tracks) and with 35 rhinos represented in test tracks, we had 64 test tracks with 2080 interactions, of which 15 were misclassified, giving an accuracy of $99.3 \%$. The predicted census estimate in this case was 32 ind., resulting in a census accuracy of $91 \%$.

As we found in Jewell et al. (2001) for black rhino, the accuracy of CPCT depended upon track size. Each of the original tracks was tested to see which other tracks it matched using the CPCT (see 'FIT methodology; FIT for censusing'), thereby determining the set structure of the library.

Fig. 3 shows the level of accuracy achieved in identifying the original tracks, using 12 variables, as a function of the number of footprints per track. For track sizes of 6 to 8 footprints the accuracy of censusing was $95 \%$ or better. The best accuracy was obtained with 
track size of 7 footprints. Not surprisingly, with short tracks, the confidence-level ellipses were larger, leading to more overlaps (which would result in an underestimate of population size in a real censusing situation), while for longer tracks the confidence-level ellipses were smaller, leading to fewer overlaps (which would result in an overestimate of population size in a real censusing situation), resulting in a reduction in accuracy in either case. Hence, the choice of track size used in this analysis is critical. Fig. 4 shows the distribution frequency of track sizes. There were 93 original tracks in the 6 to 8 footprints per track categories. However, to further increase the number of tracks which could be used in the analysis, we split or concatenated the rest of the tracks to give a total of 152 modified tracks.

\section{FIT for monitoring; CERT}

Unlike CPCT, CERT is less sensitive to track size and the number of variables used in the analysis. The 159 original tracks, which varied in length from 3 to 12 footprints, were tested in turn as described in 'FIT methodology; FIT for monitoring'. using 30 variables (using the forward stepwise procedure in JMP). Five were misclassified and one unclassified (tied) giving an accuracy of $97 \%$. Applying the second model, in which $50 \%$ of the footprints from each individual were assigned to the algorithm, of the 69 test tracks available, 3 were misclassified and 2 unclassified giving an accuracy of $93 \%$. Fig. 5 shows an example of the procedure used for CERT. Using 30 variables selected using the forward stepwise feature, canonical analysis

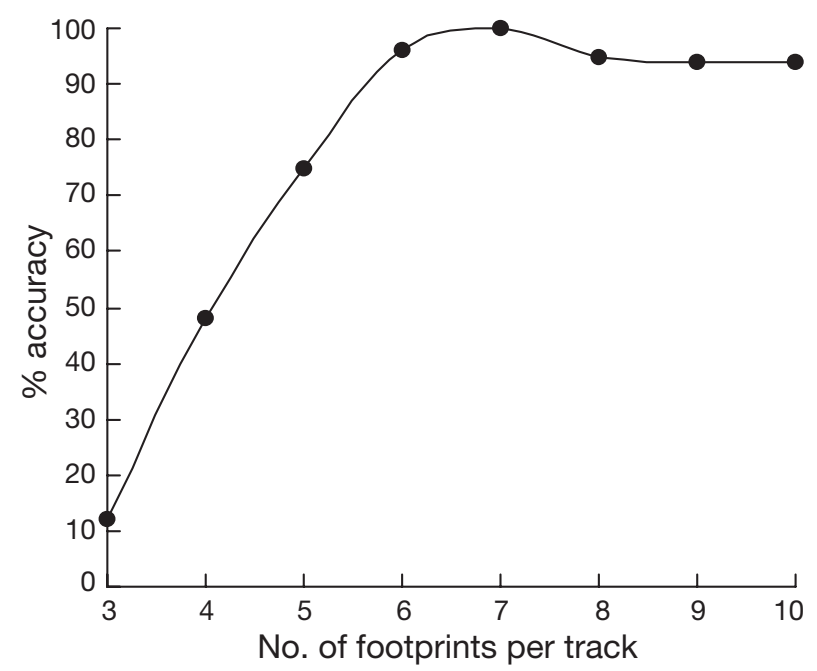

Fig. 3. Ceratotherium simum. Census accuracy using the canonical pairwise comparison technique (CPCT) as a function of the number of footprints per track produces a plot with centroids and 95\% confidence interval ellipses for each of the known 40 ind. and the 'unknown' or test track. Fig. 5a shows the outcome of the first step with the test track ellipse shown overlapping with a number of ellipses (only a section of the plot with the test track shown here). The groups whose ellipses do not overlap with the test ellipse are then eliminated and the procedure repeated using the same 30 variables (Fig. 5b). In the final stage, the ellipse furthest from the test ellipse is eliminated until the test ellipse overlaps with one other ellipse only (Fig. 5c).

\section{FIT for species identification}

For species discrimination, we used a dataset of 1636 footprints with 218 tracks, of which 59 were from black and 159 from white rhino. To test this data for track classification, we used linear LDA with 30 variables selected using the forward stepwise technique. Each track in turn was excluded in a jackknife procedure. The predicted classification for the original tracks resulted in 1 misclassified and 3 unclassified tracks (99.5\% accuracy). We further tested the robustness of the discrimination by excluding all the rest of the tracks for the same animal as the test track during the analysis. In this case 4 tracks were misclassified and 4 unclassified (98.2\% accuracy). Using the second model with a 50-50 split for the algorithm vs. test footprints, of the 118 test tracks available, one was misclassified (99.5\% accuracy). For all models the resulting classification accuracy was $>98 \%$, suggesting that FIT appeared to be highly accurate in discriminating white rhino from black rhino from their footprints.

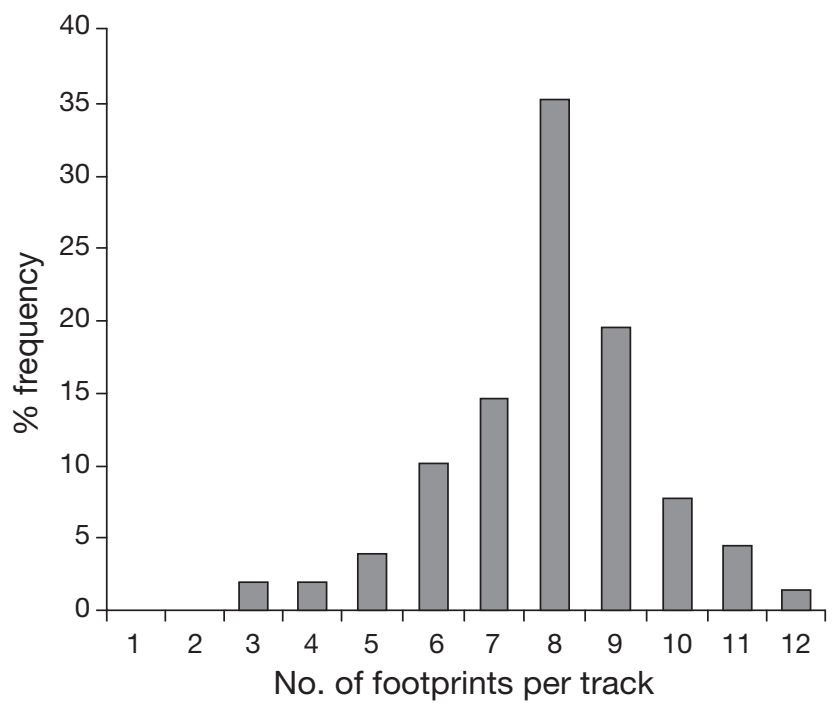

Fig. 4. Ceratotherium simum. Distribution of track size (number of footprints per track) $(\mathrm{N}=159)$ 


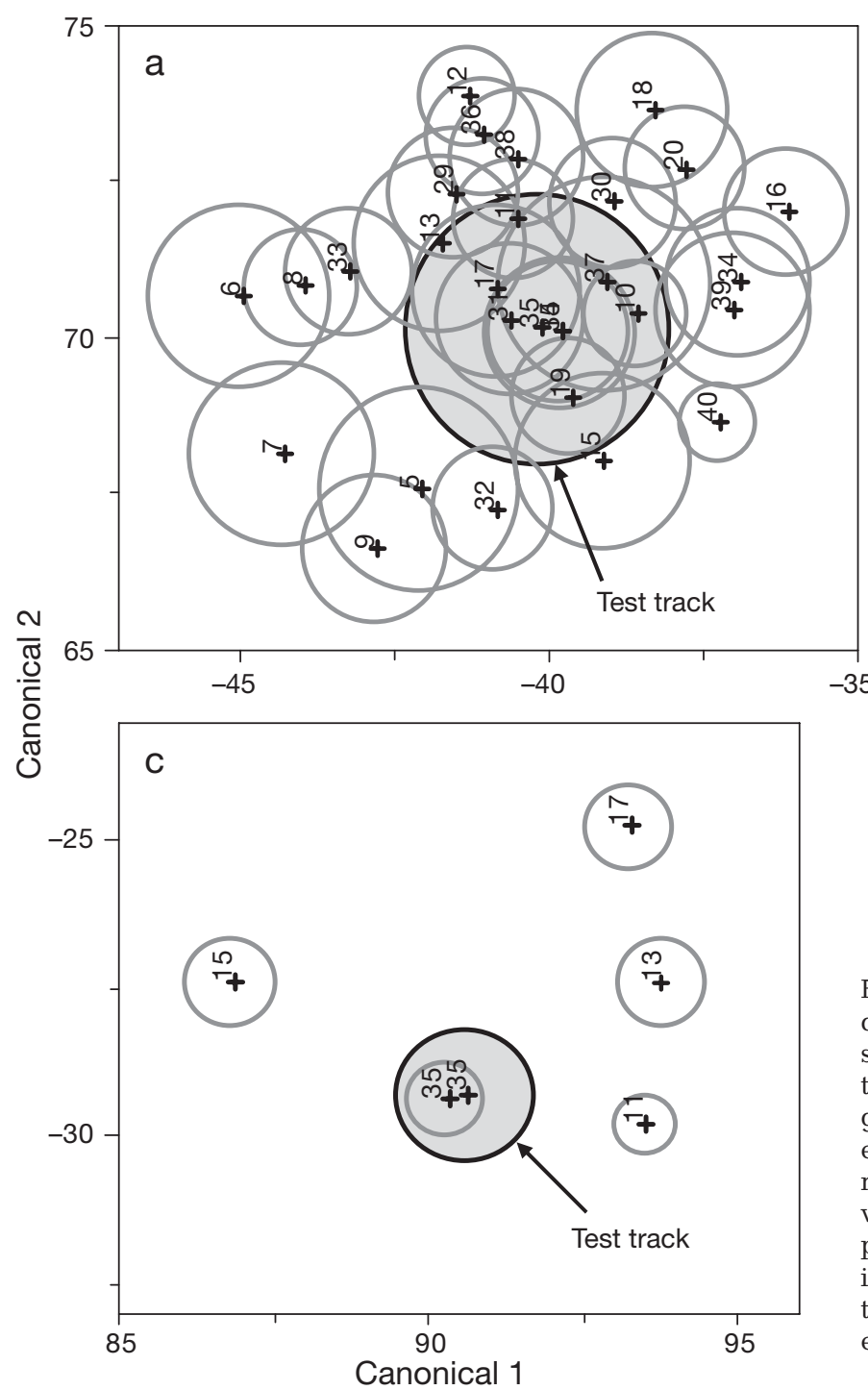

DISCUSSION

\section{Development, utility and practical application of FIT}

The ability to identify individual animals from wild populations using a cost-effective, non-invasive and accurate method would be of considerable value to population censusing and monitoring programmes (see Sharma et al. 2005). We have attempted to demonstrate the adaptability of FIT by extending and refining a previous application for black rhino (Jewell et al. 2001). We have demonstrated its implementation for white rhino and tested the accuracy of that implementation.

The FIT results for the white rhino showed that footprint identification for either censusing or monitoring could be achieved with a very high degree of accuracy. In the census scenario, both independent test models

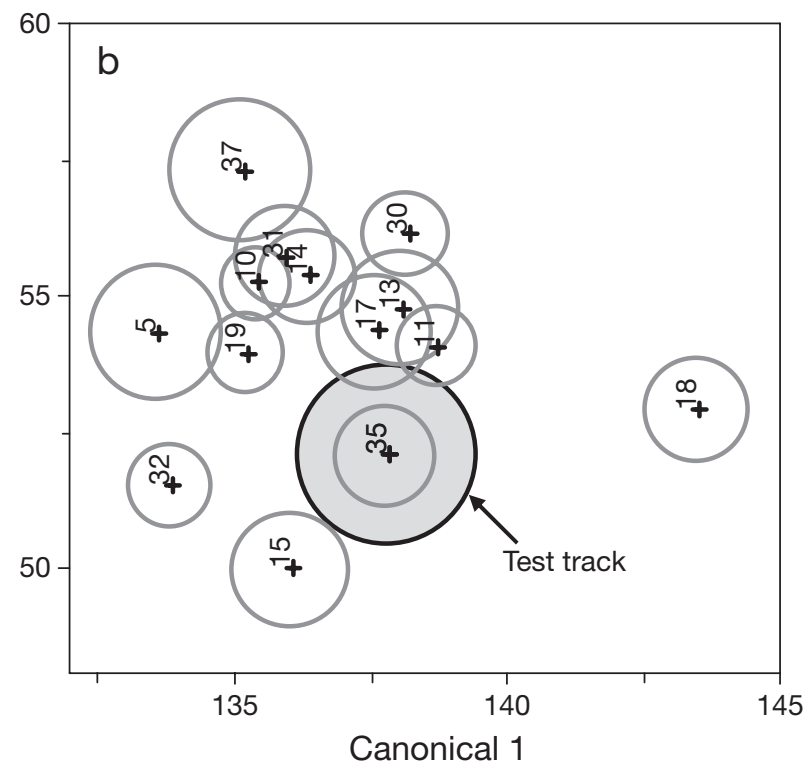

Fig. 5. (a) Plot of the first 2 canonical variates with $95 \%$ confidence ellipses and centroids for white rhino footprint measurements. In the initial phase all the 40 rhinos are included together with the test track. The test track ellipse is shown in grey overlapping with a number of ellipses. Ellipse size difference is due to sample size of rhino sets (outlying ellipses are not shown). (b) Analysis in the next phase (using the same variables) once the non-overlapping ellipses in the initial phase have been eliminated. (c) Final outcome of the analysis is when the test track ellipse overlaps with only one other. In this case the test track was from Rhino 35. The overlap of ellipses acts as the classifier for determining the predicted identity of the test track

gave high levels of accuracy with regard to the classification of pairwise matches of tracks. As a result, the predicted census estimates were close to the actual figures. However, this method is sensitive to the number of measurements used in the analysis and the number of footprints per track, and it appears that this is species-specific. Also, in order to use this method it is necessary to build the algorithm (i.e. the actual measurements and the number) from known individuals of the study species. The more individuals available for algorithm construction the better, but the trials carried out with the black and white rhinos and 2 species of tapir (S. K. Alibhai \& Z. C. Jewell unpubl. data) and the Bengal tiger (S. K. Alibhai \& Z. C. Jewell unpubl. data) suggest that 15 to 20 ind. would be sufficient (cf. Sharma et al. 2005, who recommend a minimum of 5 to 8 ind. for the Bengal tiger). To determine the number of footprints required for each individual for the initial 
algorithm construction, our data, once again, suggest that a figure of 15 to 20 would be sufficient. For example, for the present study we arbitrarily varied the number of footprints per individual from a minimum of 5 to $35+$, and tested the accuracy with which each individual footprint was classified correctly using LDA. Fig. 6 shows that the asymptote was reached at 20 footprints. We believe that this approach, i.e. using the matchability of tracks has great potential for identifying individuals of endangered species. This was supported by L. Van Bommel et al. (unpubl. data) working with the African lion Panthera leo. Using 20 footprints from each of 30 captive animals, they compared the efficacy of 2 different methods in the census scenario. They used the one-class classification approach (Tax 2001) and the canonical centroid plot method (CPCT in this study) reported by Jewell et al. (2001). They found that the canonical method gave more accurate results. Interestingly, they found that the optimal number of variables for the canonical plot was 11. Furthermore, L. Van Bommel et al. (unpubl. data) reported that because of the pairwise approach, the canonical plot method was also not sensitive to the size of population under study. We found the optimal track size to be 6 to 8 footprints, compared with L. Van Bommel et al. (unpubl. data), who suggest 8 to 10 for the African lion, and Sharma et. al (2005), who found that 4 to 12 pugmarks per track gave accuracies of between 90 and $100 \%$.

For monitoring, a new multivariate statistical discriminator, CERT, also gave very high classification accuracies of assigning the test tracks correctly. Once again, this was tested using 2 independent models. The reason for developing this technique was that our data on black rhino (Jewell et al. 2001) had shown that

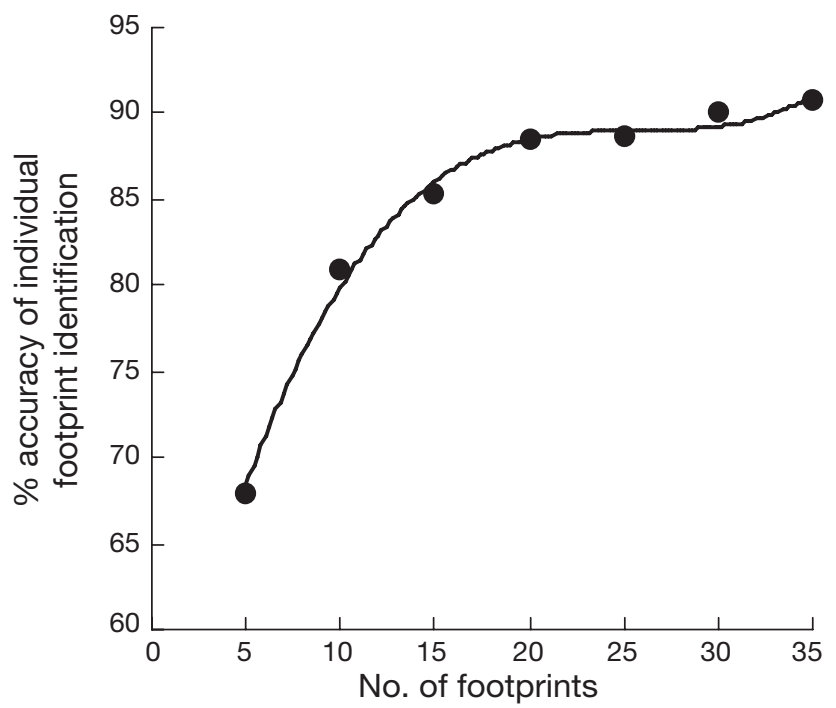

Fig. 6. Accuracy of identification of footprints as a function of number of footprints defining groups using LDA, which provided a classification based on correct/incorrect match for individual footprint, we had many unclassified tracks (i.e. equal numbers of footprints in the test track being assigned to 2 or more individuals). Using CERT ensured that the number of ties was reduced (only a single tie for 1 model and 2 ties for the other model in the present study).

One of the criticisms levelled at the use of footprints for censusing/monitoring populations is that the study/ test populations have tended to be small, therefore not representative of populations/subpopulations in the wild. The present study population of 40, with 159 tracks and 1276 footprints, is relatively large and thus provides a much more robust test for the use of footprints in individual animal identification. Interestingly, Sharma et al. (2005), who had 19 tigers and 23 pugmark-sets (tracks) in their study, make the point that the actual number of tracks which would need to be compared in any one session during a tiger census in a particular area would be 10 to 35, even in the high tiger density areas. Whilst this may be true for tigers, our data for 2 species of rhino and 2 species of tapir to date suggest that many more tracks are likely to be encountered depending, of course, on the size and distribution of the population.

We believe that FIT can be used very effectively for censusing and monitoring populations of endangered species. In their thorough review, Karanth et al. (2003) present very cogent and convincing arguments to explain why the 'pugmark' method has failed to reliably census the tiger population in India. While they consider this to be due to a paradigm failure, we suggest that this has been partly due to the lack of objectivity in the 'pugmark' method used for generations in Indian tiger censusing, and also the use of incorrect sampling methods. We believe that footprints do contain very useful information which, if extracted objectively, can be used to identify individuals accurately, and that efforts should be directed towards changing the method of using 'pugmarks' rather than dismissing their validity altogether. Our data show that it is possible to get reliable figures for absolute abundance for certain species in certain locales using FIT, and that this method can be adapted to each unique situation. For example, to obtain a census figure for the black rhino population of the Namutoni region of Etosha National Park in Namibia, we designed the sampling of the footprint collection based on the fact that there were only a limited number of water holes and the fact that the animals had to drink at least once every 24 to $48 \mathrm{~h}$ from one of these (see Owen-Smith 1988). By sampling the water holes on several occasions we ensured the collection of footprints from all the individuals in that area (Alibhai \& Jewell 1997). 
Like all censusing and monitoring techniques FIT has strengths and limitations.

The strengths are clear; it is cost effective, particularly compared with those techniques which require expensive equipment and/or veterinary input; it utilises the expertise of local trackers, who are often employed as scouts or in anti-poaching patrols in local conservation efforts; it is arguably less subjective than other commonly used monitoring techniques which rely on one form or other of subjective visual appraisal; it is not restricted in range by costly apparatus or temporal concerns, and is thus potentially able to provide a more realistic coverage of home-range than standard (non-GPS) telemetry methods or camera-trapping, and, as importantly as all the above, it is non-invasive to the species under investigation.

There are also practical limitations of FIT. The first is the temporary impact of rain (or on occasion, high wind) on the collection of usable footprints. Heavy rain generally led to delays in new footprint collection of 2 to $3 \mathrm{~d}$. With showers or lighter rain, it was often possible to find footprints the next day. Another limitation is that footprints change over time. Obviously subadult animal footprints grow, but our preliminary data suggest that even adult rhino footprints change over time. For this reason FIT used for monitoring (but obviously not censusing) is best undertaken as an ongoing routine, not punctuated by long breaks. The third limitation in implementation is, of course, the necessity for a suitable substrate and target species with a footprint of sufficient complexity; FIT is not suitable for all species and all environments, although we are currently investigating the use of FIT in more challenging substrates, such as snow and mud.

Because it requires minimal equipment, and because images can often be collected as part of general monitoring duties (e.g. anti-poaching), FIT may be an ideal monitoring tool as a first-line enquiry, or as a costeffective and objective additional tool in a monitoring armoury (the adoption of several different techniques being increasingly employed in conservation monitoring), and reflecting the gains inherent in a combination-approach.

\section{Future development of FIT}

We are adapting FIT for other endangered species. A provisional algorithm for the Bengal tiger has been developed from captive animals giving a high degree of accuracy in identifying at the individual, sex and laterality levels. We have developed algorithms for the lowland and Baird's tapirs, which also provide high accuracy of identification at the individual and species level, and are currently undertaking trials of FIT with other species, substrates and monitoring techniques.
Acknowledgements. We are grateful to the Ministry of Environment and Tourism of Namibia for providing permits for this work. We thank Juergen Matthaei, Peter and Elke Reinhardt, members of the Otjiwa Anti-poaching Unit and other staff at Otjiwa for their support. We also thank an anonymous donor for his generous support, JMP Software USA, SAS Software UK and USA, and Jennie Kiesling for providing funding and technical support. We thank 4 independent reviewers for their constructive criticism in the preparation of earlier drafts of this manuscript.

\section{LITERATURE CITED}

Alibhai SK, Jewell ZC (1997) Black rhino censusing and monitoring using a spoor recognition technique. A preliminary feasibility study for use in Etosha National Park. Report to the Ministry of Environment \& Tourism, Namibia

Alibhai SK, Jewell ZC (2001) Hot under the collar: the failure of radio-collars on black rhino (Diceros bicornis). Oryx 35:284-288

Alibhai SK, Jewell ZC (2002) Response to Atkinson, du Toit, Radcliffe, Dooley and Kock. In: Boyd IL (ed) The cost of information: should black rhinos be immobilized? J Zool (Lond) 258:279-280

Alibhai SK, Jewell ZC, Towindo SS (2001) The effects of immobilisation on fertility in female black rhino (Diceros bicornis). J Zool (Lond) 253:333-345

Amin R, Thomas K, Emslie RH, Foose TJ, van Strien N (2006) An overview of the conservation status of and threats to rhinoceros species in the wild. Int Zoo Yearb 40:96-117

Arzoumanian Z, Holmberg J, Norman B (2005) An astronomical pattern-matching algorithm for computer-aided identification of whale sharks Rhincodon typus. J Appl Ecol 42:999-1011

Caughley G, Sinclair ARE (1994) Wildlife ecology and management. Blackwell Science, Cambridge, MA

Emslie RH, Brooks M (2002) How many southern white rhinos were there? A response to Kees Rookmaaker. Pachyderm 33:100-101

Emslie RH, Milledge S, Brooks M, van Strien N, Dublin H (compilers) (2007) African and Asian rhinoceroses - status, conservation and trade. Report from the IUCN Species Survival Commission (SSC) African and Asian Rhino Specialist groups and TRAFFIC to the CITES Secretariat. Report to CITES 14th meeting, The Hague, June 2007. CoP14 Doc. 54 Annex 1:6-22

Goddard J (1966) Mating and courtship of the black rhinoceros (Diceros bicornis L.). E Afr Wildl J 4:69-75

Grigione MM, Burman P, Bleich VC, Pierce BM (1999) Identifying individual mountain lions Felis concolor by their tracks: refinement of an innovative technique. Biol Conserv 88:25-32

- Herzog CJ, Kays RW, Ray JC, Gompper ME, Zielinski WJ, Higgins R, Tymeson M (2007) Using patterns in trackplate footprints to identify individual fishers. J Wildl Manag 71:955-963

Jewell ZC, Alibhai SK, Law PR (2001) Censusing and monitoring black rhino (Diceros bicornis) using an objective spoor (footprint) identification technique. J Zool (Lond) 254:1-16

Johnson RA, Wichern DW (1998) Applied multivariate statistical analysis 4th edn. Prentice Hall, Upper Saddle River, NJ

Joubert E (1996) On the clover trail: the plight of the world rhinos. Gamsberg MacMillan, Windhoek

Karanth KU (1995) Estimating tiger Panthera tigris populations from camera-trap data using capture-recapture models. Biol Conserv 71:333-338 
Karanth KU, Nichols JD (1998) Estimation of tiger densities in India using photographic captures and recaptures. Ecology 79:2852-2862

Karanth KU, Nichols JD, Seidensticker J, Dinerstein E and others (2003) Science deficiency in conservation practice: the monitoring of tiger populations in India. Anim Conserv 6:141-146

Katona SK, Baxter B, Brazier O, Kraus S, Perkins J, Whitehead $H$ (1979) Identification of humpback whales by fluke photographs. In: Winn HE, Olla BA (eds) Behavior of marine mammals: Current perspectives in research. Vol 3 Cetaceans. Plenum Press, New York, p 33-44

Kelly MJ (2001) Computer-aided photographic matching in studies using individual identification: an example from Serengeti cheetahs. J Mammal 82:440-449

Mardia KV, Kent JT, Bibby JM (1979) Multivariate analysis. Academic Press, London

Miththapala S, Seidensticker J, Phillips LG, Fernando SBU, Smallwood JA (1989) Identification of individual leopards (Panthera pardus kotiya) using spot pattern variation. J Zool (Lond) 218:527-536

Moorhouse TP, MacDonald DW (2005) Indirect negative impacts of radio-collaring: sex ratio variation in water voles. J Appl Ecol 42:91-98

Mukinya JG (1973) Density distribution, population structure and social organization of the black rhinoceros in Masai Mara game Reserve. E Afr Wildl J 11:385-400

Murray DL, Fuller TK (2000) A critical review of the effects of marking on the biology of vertebrates, in research techniques in animal ecology: controversies and consequences. In: Boitani L, Fuller TK (eds) Columbia University Press, New York, p 15-64

Editorial responsibility: Dave Hodgson,

University of Exeter, Cornwall Campus, UK
Owen-Smith RN (1988) Megaherbivores. Cambridge University Press, Cambridge

Pennycuick CJ, Rudnai J (1970) A method of identifying individual lions Panthera leo with an analysis of the reliability of identification. J Zool 160:497-508

Riordan P (1998) Unsupervised recognition of individual tigers and snow leopards from their footprints. Anim Conserv 1:253-262

Rookmaker K (2002) Miscounted population of the southern white rhinoceros (Ceratotherium simum simum) in the early 19th Century? Pachyderm 32:22-28

Sharma S, Jhala Y, Sawarkar VB (2005) Identification of individual tigers (Panthera tigris) from their pugmarks. J Zool (Lond) 267:9-18

Stander PE, Gau //, Tsisaba D, aOma //, Ui I.I (1997) Tracking and the identification of spoor: a scientifically sound method in ecology. J Zool (Lond) 242:329-341

Tax DMJ (2001). One-class classification; concept-learning in the absence of counter-examples. Delft University of Technology, Delft

van Tienhoven AM, den Hartog JE, Reijns RA, Peddemors VM (2007) A computer-aided program for pattern matching of natural marks on the spotted raggedtooth shark Carcharias taurus. J Appl Ecol 44:273-280

Whitaker JS (1997) Use of stepwise methodology in discriminant analysis. Paper presented at the annual meeting of the Southwest Educational Research Association, Austin, TX, January 1997. Available at http://ericae.net/ft/tamu/ STEPWIS.htm

Williams BK (1982) A simple demonstration of the relationship between classification and canonical variates analysis. Am Stat 36:363-365

Submitted: August 6, 2007; Accepted: November 1, 2007 Proofs received from author(s): December 15, 2007 